Communications in Physics, Vol. 25, No. 1 (2015), pp. 29-43

DOI:10.15625/0868-3166/25/1/0

\title{
ANOMALOUS MAGNETIC MOMENT OF MUON IN ECONOMICAL 3-3-1 MODEL
}

\author{
DINH THANH BINH, DO THI HUONG, LE THO HUE, HOANG NGOC LONG \\ Institute of Physics, Vietnam Academy of Science and Technology, \\ 10 Dao Tan, Ba Dinh, Hanoi, Vietnam
}

Received 22 October 2014

Accepted for publication 28 March 2015

\begin{abstract}
We investigate in detail one - loop contribution of new particles to the muon anomalous magnetic moment $(g-2)_{\mu}$ in the framework of the economical 3-3-1 model. The main contributions to the anomalous magnetic moment come from new neutral gauge boson $Z^{\prime}$, bilepton singly charged vector boson $Y^{ \pm}$. The contributions of new neutral/charged scalars are small and can be neglected. The result is extended to other versions of 3-3-1 models. Comparing the anomalous magnetic moment value in this model with experimental value we set the extension requirement for the model.
\end{abstract}

Keywords: muon, anomalous magnetic moment, precision tests.

\section{INTRODUCTION}

At present, the anomalous magnetic moment of the muon (AMMM) gains special interest due to a hint of new physics. With the discrepancy $a_{\mu}^{e x p}-a_{\mu}^{S M}=(288 \pm 80) \times 10^{-11}$ or $3.6 \sigma$ level [1], the muon anomalous magnetic moment is currently one of the most compelling inconsistencies between data and the Standard Model (SM) predictions in all of particle physics. Facing with this deviation, one could consider the difference coming from sources such as theoretical uncertainty or contribution of new physics. Efforts in both the experimental and theoretical fronts are taking to improve the precision of both the measured value and the SM prediction.

Since the $S U(3)_{C}$ is conserved and the $S U(2)_{L}$ is usually extended in models beyond the Standard Model, one can divide the contribution to $\Delta a_{\mu}$ into two parts: hadronic and non hadronic parts. The non-hadronic part includes the electromagnetic (QED) corrections and electroweak $(\mathrm{EW})$ corrections. The QED part includes all $\gamma$ and leptonic $(e, \mu, \tau)$ loops, and the EW involves $W^{ \pm}, Z$ and Higgs. The hadronic contributions come from $S U(3)_{C}$. The hadronic corrections give main theoretical uncertainties. However, the improvement in lattice QCD and hadronic data [2] will reduce those uncertainties. If one uses $\tau$ data in the hadronic contributions [1] then this $3.6 \sigma$ deviation is reduced to $2.4 \sigma$.

This discrepancy from the expectations of the SM has an inspiration for many models of new physics, and has been used to constrain or motivate parameter values for many others, especially supersymmetric models [3]. Among new physics models, there is a class of

(C)2015 Vietnam Academy of Science and Technology 
$S U(2)_{L} \rightarrow S U(3)_{L}$ extension models of the SM have been studied in literature [4,5]. Particularly, the $a_{\mu}$ anomaly has also been investigated in the context of 3-3-1 models $[6,7]$. In this paper we will focus on the 3-3-1 model with two Higgs triplets or the economical 3-3-1 model (E331) [5]. In addition to having fewer matter fields, this model also features neutral/charged bilepton vector bosons and neutral/charged scalar bosons. The scalar sector is minimal with two Higgs triplets. These features lead to new interesting phenomenology. In this work, we aim to investigate the $a_{\mu}$ anomaly in the framework of the E331 model. We will analyze all one-loop contributions coming from new particles in the E331 model and draw our conclusions based on the total contribution.

The paper is organized as follows: in Sec. II we briefly introduce the model. Sec. III discusses the $a_{\mu}$ discrepancy from the SM, and in Sec. IV we present the $a_{\mu}$ predictions of the E331 model. Lastly, in Sec. V we draw our conclusions.

\section{THE MODEL}

The economical 3-3-1 model or the E331 model has been studied in detail in [5]. In this paper we will briefly go to features of the model for the purpose of this work. The particle content in this model which is anomaly free, is given as follows:

$$
\begin{aligned}
\psi_{i L} & =\left(\begin{array}{lll}
v_{i} & e_{i} & v_{i}^{c}
\end{array}\right)_{L}^{T} \sim\left(3,-\frac{1}{3}\right), e_{i R} \sim(1,-1), i=1,2,3 \\
Q_{1 L} & =\left(\begin{array}{lll}
u_{1} & d_{1} & U
\end{array}\right)_{L}^{T} \sim\left(3, \frac{1}{3}\right), Q_{\alpha L}=\left(\begin{array}{lll}
d_{\alpha} & -u_{\alpha} & \left.D_{\alpha}\right)_{L}^{T} \sim\left(3^{*}, 0\right), \alpha=2,3, \\
u_{i R} & \sim\left(1, \frac{2}{3}\right), d_{i R} \sim\left(1,-\frac{1}{3}\right), U_{R} \sim\left(1, \frac{2}{3}\right), D_{\alpha R} \sim\left(1,-\frac{1}{3}\right) .
\end{array}\right.
\end{aligned}
$$

The values in the parentheses denote quantum numbers based on the $\left(\mathrm{SU}(3)_{L}, \mathrm{U}(1)_{X}\right)$ symmetry.

Electric charges of the exotic quarks $U$ and $D_{\alpha}$ are the same as those of the usual quarks, i.e., $q_{U}=\frac{2}{3}$ and $q_{D_{\alpha}}=-\frac{1}{3}$.

The $\mathrm{SU}(3)_{L} \otimes \mathrm{U}(1)_{X}$ gauge group is broken spontaneously via two steps. In the first step, it is embedded in that of the SM via a Higgs scalar triplet

$$
\chi=\left(\begin{array}{lll}
\chi_{1}^{0} & \chi_{2}^{-} \quad \chi_{3}^{0}
\end{array}\right)^{T} \sim\left(3,-\frac{1}{3}\right)
$$

acquired with VEV given by

$$
\langle\chi\rangle=\frac{1}{\sqrt{2}}\left(\begin{array}{lll}
u & 0 & \omega
\end{array}\right)^{T} .
$$

In the last step, to embed the gauge group of the $\mathrm{SM}$ in $\mathrm{U}(1)_{Q}$, another Higgs scalar triplet

$$
\phi=\left(\begin{array}{lll}
\phi_{1}^{+} & \phi_{2}^{0} & \phi_{3}^{+}
\end{array}\right)^{T} \sim\left(3, \frac{2}{3}\right)
$$

is needed with the VEV as follows

$$
\langle\phi\rangle=\frac{1}{\sqrt{2}}\left(\begin{array}{lll}
0 & v & 0
\end{array}\right)^{T} .
$$


The Yukawa interactions which induce masses for the fermions can be written in the most general form as

$$
\mathscr{L}_{Y}=\left(\mathscr{L}_{Y}^{\chi}+\mathscr{L}_{Y}^{\phi}\right)+\mathscr{L}_{Y}^{\text {mix }}
$$

where the part involving our calculation in this work is

$$
\begin{aligned}
\left(L_{Y}^{\chi}+\mathscr{L}_{Y}^{\phi}\right) & =h_{11}^{\prime} \bar{Q}_{1 L} \chi U_{R}+h_{\alpha \beta}^{\prime} \bar{Q}_{\alpha L} \chi^{*} D_{\beta R} \\
& +h_{i j}^{e} \bar{\psi}_{i L} \phi e_{j R}+h_{i j}^{\varepsilon} \varepsilon_{p m n}\left(\bar{\psi}_{i L}^{c}\right)_{p}\left(\psi_{j L}\right)_{m}(\phi)_{n}+h_{1 i}^{d} \bar{Q}_{1 L} \phi d_{i R}+h_{\alpha i}^{d} \bar{Q}_{\alpha L} \phi^{*} u_{i R} \\
& + \text { h.c. }, \\
\mathscr{L}_{Y}^{\text {mix }} & =h_{1 i}^{u} \bar{Q}_{1 L} \chi u_{i R}+h_{\alpha i}^{u} \bar{Q}_{\alpha L} \chi^{*} d_{i R}+h_{1 \alpha}^{\prime \prime} \bar{Q}_{1 L} \phi D_{\alpha R}+h_{\alpha 1}^{\prime \prime} \bar{Q}_{\alpha L} \phi^{*} U_{R}+\text { h.c.. }
\end{aligned}
$$

The VEV $\omega$ gives mass for the exotic quarks $U$ and $D_{\alpha}, u$ gives mass for $u_{1}, d_{\alpha}$ quarks, while $v$ gives mass for $u_{\alpha}, d_{1}$ and all ordinary leptons. To provide mass for all ordinary quarks, both one-loop corrections and effective interaction are needed [8]. We would like to emphasize that the first step of symmetry breaking is done by VEV $\omega$, the second step is due to $u$ and $v$. Therefore, the VEVs in this model have to be satisfied the constraints

$$
u, v \ll \omega .
$$

\section{II.1. Lepton masses}

The charged leptons gain masses via the following Yukawa term

$$
\mathscr{L}_{Y}^{e}=h_{i j}^{e} \bar{\psi}_{i L} \phi e_{j R}+\text { h.c.. }
$$

Because the lepton flavour violating effects relating with charged leptons are very suppressed, we can suppose that the coupling constant $h_{i j}^{e}$ is diagonal in the flavour indices $i j$. The masses of the $e, \mu, \tau$ are

$$
m^{i}=\frac{h_{i i}^{e} v}{\sqrt{2}}
$$

with $i=e, \mu, \tau$.

Masses of neutrinos are discussed in detail in [9]. They are very small so throughout our calculation, they are ignored.

\section{II.2. Higgs and gauge bosons}

In this model, the most general Higgs potential has very simple form

$$
\begin{aligned}
V(\chi, \phi)= & \mu_{1}^{2} \chi^{\dagger} \chi+\mu_{2}^{2} \phi^{\dagger} \phi+\lambda_{1}\left(\chi^{\dagger} \chi\right)^{2}+\lambda_{2}\left(\phi^{\dagger} \phi\right)^{2} \\
& +\lambda_{3}\left(\chi^{\dagger} \chi\right)\left(\phi^{\dagger} \phi\right)+\lambda_{4}\left(\chi^{\dagger} \phi\right)\left(\phi^{\dagger} \chi\right) .
\end{aligned}
$$

Let us shift the Higgs around their vacuum expectation values such as

$$
\begin{aligned}
\phi^{T} & =\left(\phi_{1}^{\dagger}, \frac{1}{\sqrt{2}}\left(v+S_{2}+i A_{2}\right), \phi_{3}^{\dagger}\right), \\
\chi^{T} & =\left(\frac{1}{\sqrt{2}}\left(u+S_{1}+i A_{1}\right), \chi_{2}^{-}, \frac{1}{\sqrt{2}}\left(w+S_{3}+i A_{3}\right)\right) .
\end{aligned}
$$

After symmetry breaking, all pseudo scalar degrees are Goldstone bosons eaten by gauge bosons. In the mass basis all real scalar Higgses $\left(H^{0}, H_{1}^{0}, G_{4}\right)^{T}$ relate to the original $\left(S_{1}, S_{2}, S_{3}\right)$ by a 
transformation [10]

$$
\left(\begin{array}{l}
S_{1} \\
S_{2} \\
S_{3}
\end{array}\right)=\left(\begin{array}{ccc}
-s_{\zeta} s_{\theta} & s_{\zeta} s_{\theta} & c_{\theta} \\
c_{\zeta} & s_{\zeta} & 0 \\
s_{\zeta} c_{\theta} & c_{\zeta} c_{\theta} & -s_{\theta}
\end{array}\right)\left(\begin{array}{c}
H^{0} \\
H_{1}^{0} \\
G_{4}
\end{array}\right)
$$

where

$$
t_{2 \zeta}=\tan 2 \zeta \equiv \frac{\lambda_{3} m_{W} m_{X}}{\lambda_{1} m_{X}^{2}-\lambda_{2} m_{W}^{2}},
$$

$c_{\zeta}=\cos \zeta, s_{\zeta}=\sin \zeta, t_{\theta}=\tan \theta=\frac{u}{w}, c_{\theta}=\cos \theta$ and $s_{\theta}=\sin \theta$. In our calculation of AMMM we will assume that the couplings $\lambda_{1}, \lambda_{2}, \lambda_{3}$ are the same order therefore $\tan 2 \zeta$ is of order $\frac{m_{W}}{m_{X}}$ and $\sin \zeta \simeq \frac{m_{W}}{2 m_{X}}$.

The Goldstone boson $G_{4}$ is eaten by $X^{0}$ boson. Masses of two other physical Higgses are

$$
m_{H_{0}, H_{0}^{1}}^{2}=\lambda_{2} v^{2}+\lambda_{1}\left(u^{2}+w^{2}\right)-\sqrt{\left[\lambda_{2} v^{2}-\lambda_{1}\left(u^{2}+w^{2}\right)\right]^{2}+\lambda_{3} v^{2}\left(u^{2}+w^{2}\right)} .
$$

In the limit $u, v \ll w$, we get $m_{H^{0}}^{2} \simeq \frac{\left(4 \lambda_{1} \lambda_{2}-\lambda_{3}^{2}\right) v^{2}}{2 \lambda_{1}}$ and $m_{H_{1}^{0}}^{2} \simeq 2 \lambda_{2} w^{2}$. Hence, the $H^{0}$ is normally identified with the SM Higgs.

In the charged Higgs sector, there is only one physical charged Higgs, namely $\mathrm{H}_{2}^{+}$with mass eigenstate given as

$$
H_{2}^{+}=\frac{1}{\sqrt{u^{2}+v^{2}+w^{2}}}\left(u \phi_{1}^{\dagger}+v \chi_{2}^{\dagger}+w \phi_{3}^{\dagger}\right) .
$$

In the limit $u, v \ll w$, the Higgs physical states are given in [5] as

$$
H^{o} \simeq S_{2}, \quad H_{1}^{o} \simeq S_{3}, \quad H_{2}^{+} \simeq \phi_{3}^{+} .
$$

Lagrangian containing Higgs-lepton-lepton couplings is

$$
\mathscr{L}_{Y}^{l}=2 h_{a b}^{v}\left(\bar{v}_{a L}^{c} l_{b L} \phi_{3}^{+}-\bar{v}_{a R} l_{b L} \phi_{1}^{+}\right)+h_{a a}^{l}\left(\bar{v}_{a L} l_{a R} \phi_{1}^{+}+\bar{v}_{a R}^{c} l_{a R} \phi_{3}^{+}+\bar{l}_{a L} l_{a R} \phi_{2}^{0}\right)+\text { H.c.. }
$$

We can ignore the first term in (10)with $h^{v}$ being proportional to neutrino masses which are very suppressed [9]. Note that $h_{\mu}^{l}=\frac{\sqrt{2} m_{\mu}}{v}$. Couplings of Higgses and $\mu$ are represented in Table 1 .

Table 1. Higgs-lepton-lepton vertices

\begin{tabular}{|c|c|c|c|}
\hline Vertex & Factor & Vertex & Factor \\
\hline $\bar{\mu} \mu H^{0}$ & $\frac{i \sqrt{2} m_{\mu} c_{\zeta}}{v}$ & $\bar{\mu} \mu H_{1}^{0}$ & $\frac{i \sqrt{2} m_{\mu} s_{\zeta}}{v}$ \\
$\bar{v}_{\mu} \mu H_{2}^{+}$ & $\frac{i \sqrt{2} m_{\mu} w s_{\theta}}{v \sqrt{w^{2}+c_{\theta}^{2} v^{2}}} P_{R}$ & $\bar{v}_{\mu}^{c} \mu H_{2}^{+}$ & $\frac{i \sqrt{2} m_{\mu} w c_{\theta}}{v \sqrt{w^{2}+c_{\theta}^{2} v^{2}}} P_{R}$ \\
\hline
\end{tabular}

We can quickly estimate that the couplings of both neutral and charged scalars in the E331 model are of order $10^{-3}$. Hence we expect the contribution of neutral and charged Higgs is small. 
The gauge bosons gain their masses due to this symmetry breaking. The gauge boson mass spectrum is given in [5] as follows:

$$
\begin{aligned}
m_{W^{ \pm}}^{2} & =\frac{g^{2} v^{2}}{4}, \quad m_{Y^{ \pm}}^{2}=\frac{g^{2}}{4}\left(u^{2}+v^{2}+\omega^{2}\right), \quad m_{X}^{2}=\frac{g^{2}}{4}\left(w^{2}+u^{2}\right), \\
m_{Z}^{2} & \simeq \frac{g^{2}}{4 c_{w}^{2}}\left(v^{2}-3 u^{2}\right), \quad m_{Z^{\prime}}^{2} \simeq \frac{g^{2} c_{w}^{2} \omega^{2}}{3-4 s_{w}^{2}},
\end{aligned}
$$

where $s_{w}=\sin \theta_{w}$ and $c_{w}=\cos \theta_{w} . Z$ and $Z^{\prime}$ correspond to $Z^{1}$ and $Z^{2}$ in Ref. [5]. Because $u \ll v \ll w$, we can get

$$
m_{Z^{\prime}}^{2}=\frac{4 c_{W}^{2}}{3-4 s_{W}^{2}}\left(m_{Y}^{2}-m_{W}^{2}\right)
$$

\section{II.3. Charged currents}

The interactions between the charged gauge bosons and fermions can be written in the form [10]

$$
H^{\mathrm{CC}}=\frac{g}{\sqrt{2}}\left(J_{W}^{\mu-} W_{\mu}^{+}+J_{Y}^{\mu-} Y_{\mu}^{+}+J_{X}^{\mu 0 *} X_{\mu}^{0}+\text { h.c. }\right),
$$

where all parts used in this work are

$$
\begin{aligned}
& J_{W}^{\mu-}=c_{\theta} \bar{v}_{i L} \gamma^{\mu} e_{i L}+s_{\theta} \bar{v}_{i L}^{c} \gamma^{\mu} e_{i L}=\frac{1}{2}\left[c_{\theta} \bar{v}_{i} \gamma^{\mu}\left(1-\gamma_{5}\right) e_{i}+s_{\theta} \bar{v}_{i}^{c} \gamma^{\mu} \gamma^{\mu}\left(1-\gamma_{5}\right) e_{i}\right], \\
& J_{Y}^{\mu-}=c_{\theta} \bar{v}_{i L}^{c} \gamma^{\mu} e_{i L}-s_{\theta} \bar{v}_{i L} \gamma^{\mu} e_{i L}=\frac{1}{2}\left[c_{\theta} \bar{v}_{i}^{c} \gamma^{\mu}\left(1-\gamma_{5}\right) e_{i}-s_{\theta} \bar{v}_{i} \gamma^{\mu}\left(1-\gamma_{5}\right) e_{i}\right] .
\end{aligned}
$$

The $X^{0}$ boson does not couple with charged leptons so it does not contribute to the $a_{\mu}$. The limit of $u \ll v, w$ implies that $\theta \simeq 0$ with $\tan \theta \equiv u / w$. So the current of the $W$ boson relating with neutrino and contribution of this current are nearly the same as those in the SM limit. In contrast, the main contribution of $Y \pm$ boson to $a_{\mu}$ is the one relating with neutrinos beyond the SM, $v^{c}$. The details computation of these contributions will be investigated in the following sections.

\section{II.4. Neutral currents}

Interactions of the neutral currents with fermions have a common form

$$
\mathscr{L}^{\mathrm{NC}}=\frac{g}{2 c_{W}} \bar{f} \gamma^{\mu}\left[g_{k V}(f)-g_{k A}(f) \gamma^{5}\right] f Z_{\mu}^{k}, k=Z, Z^{\prime}
$$

The detail for coupling constant is given in [5]. In the limit of $u \ll v, w$ we have

$$
\begin{array}{ll}
g_{V}^{Z} \simeq \frac{4 s_{W}^{2}-1}{2}\left[c_{\varphi}+\frac{s_{\varphi}}{\sqrt{3-4 s_{W}^{2}}}\right], \quad g_{A}^{Z} \simeq-\frac{1}{2}\left[c_{\varphi}+\frac{s_{\varphi}}{\sqrt{3-4 s_{W}^{2}}}\right], \\
g_{V}^{Z^{\prime}} \simeq \quad \frac{4 s_{W}^{2}-1}{2}\left[-\frac{c_{\varphi}}{\sqrt{3-4 s_{W}^{2}}}+s_{\varphi}\right], \quad g_{A}^{Z^{\prime}} \simeq-\frac{1}{2}\left[-\frac{c_{\varphi}}{\sqrt{3-4 s_{W}^{2}}}+s_{\varphi}\right],
\end{array}
$$

where $t_{2 \varphi} \simeq-\frac{\sqrt{3-4 s_{w}^{2}} v^{2}}{2 c_{w}^{4} \omega^{2}}$. In the limit of $v \ll w$, i.e., $s_{\varphi} \simeq 0$, the couplings of the $Z$ boson to leptons in (16) are also the same as those in the SM. Anyway, contributions of the $Z$ boson to $a_{\mu}$ will be 
different from that of the SM if $w$ is small enough. In the below computation, we will not ignore $\varphi$ value.

\section{THE ANOMALOUS MAGNETIC MOMENT OF THE MUON}

As charged elementary particle, the AMMM is related to its intrinsic spin by the gyromagnetic ratio $g_{\mu}$ :

$$
\vec{\mu}_{\mu}=g_{\mu}\left(\frac{q}{2 m}\right) \vec{S}
$$

where $g_{\mu}$, within the framework of the Dirac equation, is expected to be equal to two for a structureless spin $1 / 2$ particle. However, quantum loop corrections lead to a deviation from this value which are parametrized in terms of $a_{\mu}=\left(g_{\mu}-2\right) / 2$. Up to now the contribution from physics of SM to $a_{\mu}$ is generally divided into three parts:

$$
a_{\mu}^{S M}=a_{\mu}^{Q E D}+a_{\mu}^{E W}+a_{\mu}^{H a d} .
$$

The QED part includes all photonic and leptonic $(e, \mu, \tau)$ loops starting with the classic $\frac{\alpha}{2 \pi}$ contribution. QED contribution has been computed through 4 loops and estimated at the 5-loop level [11]. Determined in $[11,12]$ leads to

$$
a_{\mu}^{Q E D}=116584718.95(0.08) \times 10^{-11} .
$$

Loops involving heavy $W^{ \pm}, Z, H$ give contributions to $a_{\mu}^{E W}$. These contributions are suppressed by at least a factor of $\frac{\alpha}{\pi} \frac{m_{\mu}^{2}}{m_{W}^{2}} \simeq 4 \times 10^{-9}$. At the one-loop level [13] $a_{\mu}^{E W}=194.8 \times 10^{-11}$. Two loops contributions are relatively large and negative [14] while 3-loops leading logarithms are negligible [14] resulting the total

$$
a_{\mu}^{E W}=153.6(1.0) \times 10^{-11} .
$$

The hadronic (quark and gluon) loop contributions give the rise to theoretical uncertainties. The hadronic vacuum polarization is calculated and inferred either from $e^{+} e^{-} \rightarrow$ hadrons or $\tau \rightarrow$ hadrons data [1]. The next largest uncertainty is associated with hadronic light-by-light scattering, which cannot, at present, be determined from data, but rather must be calculated using hadronic models that correctly reproduce the properties of QCD. Motivated by large- $N_{C}$ QCD [15] $a_{\mu}^{\mathrm{Had}}[N L O]$ is found to be

$$
a_{\mu}^{\mathrm{Had}}[N L O]=7(26) \times 10^{-11} .
$$

Sum up all the contribution above we have the SM prediction

$$
a_{\mu}^{S M}=116591803(1)(42)(26) \times 10^{-11} .
$$

Recently, the E821 experiment has measured [16] and take into account correlations between systematic uncertainties one finds

$$
\begin{aligned}
a_{\mu}^{E 821} & =116592091(54)(33) \times 10^{-11} \\
& =(116592091 \pm 63.3) \times 10^{-11} .
\end{aligned}
$$

Hence,

$$
\begin{aligned}
\Delta a_{\mu}(E 821-S M) & =288(63)(49) \times 10^{-11} \\
& =(288 \pm 80) \times 10^{-11}
\end{aligned}
$$


This difference is about $3.6 \sigma$. The present theoretical error is $\pm 49.4 \times 10^{-11}$. It has been suggested that uncertainty on the lowest-order hadronic contribution could be reduced to $25 \times$ $10^{-11}$ with existing data and further work on the hadronic light-by-light corrections could reduce the total SM error to as little as $\pm 30 \times 10^{-11}[17,18]$. With the proposed experimental error of $\pm 16 \times 10^{-11}$ for the experiment with improved statistics at Fermilab, the combined uncertainty for the difference between theory and experiment might reach $\pm 34 \times 10^{-11}$, better by a factor $\sim 2.4 \sigma$ than the current error [17].

\section{CONTRIBUTIONS TO THE $(g-2)_{\mu}$}

In this section we will investigate the implications of the anomaly in $a_{\mu}$ from the perspective of the E331 model. The new particles give the contribution to the AMMM at first order are the new charged gauge bosons $Y^{ \pm}, Z^{\prime}$ and new scalars $H^{0}, H_{2}^{+}$. We will explore each new boson's contributions independently. The constraints on the new boson masses are based on the sum of all the contributions. Apart from the contribution of the SM, in the E331 model new contributions to $a_{\mu}$ from the particular Feynman diagrams are presented in the Fig. 1.

\section{IV.1. Singly charged vector}

Properties of neutrinos masses in the E331 model are discussed in [19]. In general, the interactions of muon to charged gauge vector boson $V^{ \pm}$have the $V-A$ form, there are two kinds of contribution to the AMMM, namely the vector and axial vector currents. Unlike the SM or minimal 331 model, the E331 model includes right-handed neutrinos, i.e., $v_{L}^{c}$ so the $W$ and $Y$ bosons also couple with this neutrino apart from the left-handed ones. All possible Feynman diagrams contributing to $a_{\mu}$ caused by these charged currents are presented in the Fig. 1(a). According to the Ref. [20], each of these contributions can be expressed by a formula,

$$
\Delta a_{\mu}^{V, A}\left(V^{ \pm}\right)=\frac{\left(f^{V, A}\right)^{2}}{8 \pi^{2}} \frac{m_{\mu}^{2}}{m_{V}^{2}} \int_{0}^{1} d x \frac{F^{V, A}(x)}{\varepsilon^{2} \lambda^{2}(1-x)\left(1-\varepsilon^{-2} x\right)+x}
$$

where $f^{V, A}$ is the coupling strength between the muon and the gauge boson $V$ with mass $m_{V}$, $\varepsilon=\frac{m_{v\left(v^{c}\right)}}{m_{\mu}}$, and $\lambda=\frac{m_{\mu}}{m_{V}}$. The function $F^{V, A}(x)$ depends on the type of coupling, in particular, for vector currents we have

$$
F_{V}(x)=2 x^{2}(1+x-2 \varepsilon)+\lambda^{2}(1-\varepsilon)^{2} \cdot x(1-x)(x+\varepsilon),
$$

and for axial vector currents

$$
F_{A}(x)=2 x^{2}(1+x+2 \varepsilon)+\lambda^{2}(1+\varepsilon)^{2} \cdot x(1-x)(x-\varepsilon) .
$$

In the framework of the E331 model, $m_{v}, m_{v^{c}} \ll m_{\mu} \ll m_{V}$ so we can take the approximation $\varepsilon, \lambda \rightarrow 0$.

In this approximation we have

$$
\Delta a_{\mu}\left(Y^{ \pm}\right)=\frac{\left[\left(f^{V}\right)^{2}+\left(f^{A}\right)^{2}\right]}{8 \pi^{2}}\left(\frac{m_{\mu}^{2}}{m_{Y}^{2}}\right)\left(\frac{5}{3}\right) .
$$


The contribution of the $W$ boson can also be calculated based on the formula (18), where the coupling strength $f_{A}^{W}=f_{V}^{W}=\frac{g}{2 \sqrt{2}} c_{\theta}$ for $v$ and $f_{A}^{W}=f_{V}^{W}=\frac{g}{2 \sqrt{2}} s_{\theta}$ for $v^{c}$. Hence the total contribution to $a_{\mu}$ from the $W$ boson is

$$
a_{\mu}^{E 331}(W)=a^{S M}(W) \times\left(s_{\theta}^{2}+c_{\theta}^{2}\right)=a^{S M}(W),
$$

where $a^{S M}(W)$ is the electroweak contribution of the $W$ boson to $a_{\mu}$ in the limit of the SM [21],

$$
a_{\mu}^{S M}(W)=\frac{\sqrt{2} G_{\mu} m_{\mu}^{2}}{16 \pi^{2}} \times \frac{10}{3} \simeq 388.70(0) \times 10^{-11}
$$

with $G_{\mu}=\frac{g^{2} \sqrt{2}}{8 m_{W}^{2}}$.

Regarding to the $Y$ boson corresponding to the currents are shown in (14), the coupling strengths are $f_{V}=f_{A}=\frac{g}{2 \sqrt{2}} s_{\theta}$ for $v$ and $\frac{g}{2 \sqrt{2}} c_{\theta}$ for $v^{c}$. Similarly to the case of the $W$ boson, the total contribution to $a_{\mu}$ of diagrams relating with the $Y$ boson does not depend on the $\theta$, as expressed by the following formula

$$
\Delta a_{\mu}\left(Y^{ \pm}\right)=\frac{g^{2} m_{\mu}^{2}}{32 \pi^{2} m_{Y}^{2}}\left(\frac{10}{6}\right)=a^{S M}(W) \times \frac{m_{W}^{2}}{m_{Y^{+}}^{2}} .
$$

We emphasize that our formula (20) is different from formula (39) of Ref. [7] by a factor of 4. This is because of difference in calculating the coupling strengths $f^{V, A}$ between neutrinos with charged gauge bosons. Because the electroweak contribution of charged gauge bosons to $a_{\mu}$ can be calculated by the general formula (18) for all gauge bosons including the $W$ boson in the SM frame work, it can be checked that the formula (20) is consistent with the case of the $W$ contribution in the limit $m_{Y}^{2} \rightarrow m_{W}^{2}$. The contribution of new charged gauge bosons is very important in the considering model because of the following reasons:

- They have the similar coupling strength with neutrinos so the electroweak contributions of them to $a_{\mu}$ are different from that of $W$ boson by only a factor of $m_{W}^{2} / M_{Y}^{2}$.

- The electroweak contribution of these charged gauge bosons is the largest contribution to $\Delta a_{\mu}^{N P} \equiv a_{\mu}^{e p x}-a_{\mu}^{S M}=288(80) \times 10^{-11}$. Other contributions from 3-3-1 effects are smaller at lest a factor of $10^{-1}$.

- Electroweak contribution from the $W$ boson in (19) is the same order with total contribution from new physics $\Delta a_{\mu}^{N P}$.

The above reasons imply that masses of the new charged boson are the same order with the mass of the $W$ boson, i.e., $\frac{v}{w} \sim \mathscr{O}(1)$, as values of the total contribution from E331 effects can approach to value of $a_{\mu}^{N P}$.

\section{IV.2. $Z^{\prime}$ boson}

The interactions of muon with neutral gauge bosons $Z$ and $Z^{\prime}$ are given in (15), corresponding to one-loop diagrams in the Fig. 1(d) that contribute to $a_{\mu}$. According to Ref. [20], analytic formula for the contribution of the $Z^{\prime}$ boson can be written as follows

$$
\Delta a_{\mu}\left(Z^{\prime}\right)=\frac{1}{8 \pi^{2}} \frac{m_{\mu}^{2}}{m_{Z}^{\prime 2}} \int_{0}^{1} d x \frac{\left(C_{V}^{Z^{\prime}}\right)^{2} F_{V}(x)+\left(C_{A}^{Z^{\prime}}\right)^{2} F_{A}(x)}{(1-x)\left(1-\lambda^{2} x\right)+\varepsilon^{2} \lambda^{2} x},
$$


where we have denoted

$$
\begin{aligned}
C_{V}^{Z^{\prime}} & =\frac{g}{2 c_{W}} g_{V}^{Z^{\prime}}(f), \quad C_{A}^{Z^{\prime}}=\frac{-g}{2 c_{W}} g_{A}^{Z^{\prime}}(f), \\
\varepsilon & =1, \quad \lambda=\frac{m_{\mu}}{m_{Z^{\prime}}}
\end{aligned}
$$

with $c_{W}=\cos \theta_{W}$. Factors $g_{A}^{Z^{\prime}}$ and $g_{V}^{Z^{\prime}}$ are given in (16). Specific forms of functions $F_{V}(x)$ and $F_{A}(x)$ are

$$
\begin{aligned}
& F_{V}(x)=2 x(1-x) x, \\
& F_{A}(x)=2 x(1-x)(x-4)-4 \lambda^{2} x^{3} .
\end{aligned}
$$

Let us estimate the expression (21) in the special limit given in (5). In this limit the $\theta$ is small then $g_{2 V}, g_{2 A}$ can be written roughly as given in (16). Hence, these integrals are simplified to give a contribution of the $Z^{\prime}$ to the AMMM as

$$
\begin{aligned}
\Delta a_{\mu}\left(Z^{\prime}(f)\right) & =\frac{m_{\mu}^{2}}{4 \pi^{2} m_{Z}^{\prime 2}} \frac{1}{3}\left[\left(C_{V}^{Z^{\prime}}\right)^{2}-5\left(C_{A}^{Z^{\prime}}\right)^{2}\right] \\
& =a_{\mu}^{S M}(Z(f)) \times \frac{m_{Z}^{2}}{m_{Z^{\prime}}^{2}}\left[\frac{-c_{\varphi}}{\sqrt{3-4 s_{W}^{2}}}+s_{\varphi}\right]^{2},
\end{aligned}
$$

where $a_{\mu}^{S M}(Z(f))$ is the electroweak contribution of the $Z$ boson in the SM framework, namely [21]

$$
a_{\mu}^{S M}(Z(f))=\frac{\sqrt{2} G_{\mu} m_{\mu}^{2}}{16 \pi^{2}} \times \frac{\left(-1+4 s_{W}^{2}\right)^{2}-5}{3} \simeq-193.89(2) \times 10^{-11} .
$$

Similarly, contribution of the $Z$ to the $a_{\mu}$ is

$$
\begin{aligned}
\Delta a_{\mu}(Z(f)) & =\frac{m_{\mu}^{2}}{4 \pi^{2} m_{Z}^{2}} \frac{1}{3}\left[\left(C_{V}^{Z}\right)^{2}-5\left(C_{A}^{Z}\right)^{2}\right] \\
& =a_{\mu}^{S M}(Z(f)) \times\left[c_{\varphi}+\frac{s_{\varphi}}{\sqrt{3-4 s_{W}^{2}}}\right]^{2},
\end{aligned}
$$

where $C_{A, V}^{Z}$ are defined the same as the case of the $Z^{\prime}, g_{A}^{Z}$ and $g_{V}^{Z}$ are also given in (16). Note that $g_{V}^{Z}$ and $g_{A}^{Z}$ have exactly form of those in the SM limit if $\varphi=0$ and the contribution to $a_{\mu}$ will be $\left.\Delta a_{\mu}(Z(f))\right|_{\varphi=0}=a_{\mu}^{S M}(Z(f))$. Because the values of $\varphi$ depend directly on the ratio of two symmetry breaking scales which we want to determine so it cannot be ignored. So unlike the case of the $W$ boson, there is a small deviation between the E331 model and the SM in contribution of $Z$ boson to $a_{\mu}$ if $w$ is small enough.

\section{IV.3. Neutral scalars}

The model contains only one new neutral massive Higgs $\left(H_{1}^{0}\right)$. This neutral scalar gives the contribution to AMMM. The related diagrams are shown in Fig. 1(c). 


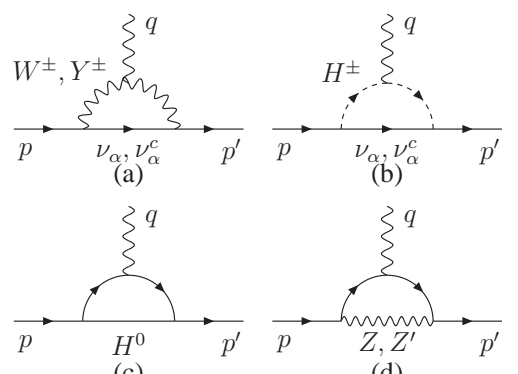

Fig. 1. Feynman diagrams contributing to the $(g-2)_{\mu}$.

The analytical expression for a real scalar Higgs contribution is presented as follows

$$
\Delta a_{\mu}\left(h^{0}\right)=\frac{f_{h^{0}}^{2}}{8 \pi^{2}} \frac{m_{\mu}^{2}}{m_{h^{0}}^{2}} \int_{0}^{1} d x \frac{x^{2}(1+\varepsilon-x)}{(1-x)\left(1-\lambda^{2} x\right)+\varepsilon^{2} \lambda^{2} x},
$$

where $\varepsilon=1, \lambda=m_{\mu} / m_{h^{0}}$.

In our case $\mu \ll m_{h^{0}}$, we get

$$
\Delta a_{\mu}\left(h^{0}\right)=\frac{f_{h^{0}}^{2}}{4 \pi^{2}} \frac{m_{\mu}^{2}}{m_{h^{0}}^{2}}\left[\ln \left(\frac{m_{h^{0}}}{m_{\mu}}\right)-\frac{7}{12}\right] .
$$

Contribution of two neutral Higgses in the E331 model is

$$
\begin{aligned}
a_{\mu}^{\mathrm{E} 331}\left(h^{0}\right) & =\frac{m_{\mu}^{2}}{4 \pi^{2} v^{2}}\left\{\frac{m_{\mu}^{2} c_{\zeta}^{2}}{m_{H^{0}}^{2}}\left[\ln \left(\frac{m_{H^{0}}}{m_{\mu}}\right)-\frac{7}{12}\right]+\frac{m_{\mu}^{2} s_{\zeta}^{2}}{m_{H_{1}^{0}}^{2}}\left[\ln \left(\frac{m_{H_{1}^{0}}}{m_{\mu}}\right)-\frac{7}{12}\right]\right\} \\
& =\frac{\sqrt{2} G_{\mu} m_{\mu}^{2}}{4 \pi^{2}}\left\{\frac{m_{\mu}^{2} c_{\zeta}^{2}}{m_{H^{0}}^{2}}\left[\ln \left(\frac{m_{H^{0}}}{m_{\mu}}\right)-\frac{7}{12}\right]+\frac{m_{\mu}^{2} s_{\zeta}^{2}}{m_{H_{1}^{0}}^{2}}\left[\ln \left(\frac{m_{H_{1}^{0}}}{m_{\mu}}\right)-\frac{7}{12}\right]\right\},
\end{aligned}
$$

where $m_{H^{0}, H_{1}^{0}}^{2}$ and $f_{H^{0}, H_{1}^{0}}$ are given in (9) and Table 1, respectively.

Since we identify $H^{0}$ with SM Higgs therefore we obtain the difference caused by the E331 model Higgs $H_{1}^{0}$.

$$
\Delta a_{\mu}^{\mathrm{E} 331}\left(H_{1}^{0}\right)=\frac{\sqrt{2} G_{\mu} m_{\mu}^{2}}{4 \pi^{2}}\left\{\frac{m_{\mu}^{2} s_{\zeta}^{2}}{m_{H_{1}^{0}}^{2}}\left[\ln \left(\frac{m_{H_{1}^{0}}}{m_{\mu}}\right)-\frac{7}{12}\right]\right\},
$$

We can approximate $\sin ^{2} \zeta \simeq \zeta^{2} / 4$ where $\zeta \simeq \frac{m_{W}}{2 m_{X}}$ if $m_{X} \gg m_{W}$. The contribution of new neutral Higgs then can be approximated as

$$
\Delta a_{\mu}^{\mathrm{E} 331}\left(H_{1}^{0}\right)=\frac{\sqrt{2} G_{\mu} m_{\mu}^{4}}{64 \pi^{2}} \frac{m_{W}^{2}}{\left(m_{Y}^{2}+m_{W}^{2}\right)} \frac{1}{m_{H_{1}^{0}}^{2}}\left[\ln \left(\frac{m_{H_{1}^{0}}}{m_{\mu}}\right)-\frac{7}{12}\right] .
$$

A roughly estimation from which we can see that the contribution of the new neutral Higgs is of order $10^{-10} \frac{1}{m_{H_{1}^{2}}^{2}}$. Hence this contribution is very small and can be neglected. 


\section{IV.4. Singly charged scalars}

Feynman diagrams are already shown in FIG. 1(b). Contributions in the general case

$$
\Delta a_{\mu}\left(h^{ \pm}\right)=\frac{f_{h^{ \pm}}^{2}}{8 \pi^{2}} \frac{m_{\mu}^{2}}{m_{h^{ \pm}}^{2}} \int_{0}^{1} d x \frac{-x(1-x)(x+\varepsilon)}{\varepsilon^{2} \lambda^{2}(1-x)\left(1-\varepsilon^{-2} x\right)+x},
$$

where $\varepsilon=m_{v, v^{c}} / m_{\mu}, \lambda=m_{\mu} / m_{h^{ \pm}}$. The limit $\varepsilon, \lambda \simeq 0$, gives [20]

$$
\Delta a_{\mu}\left(h^{ \pm}\right)=\frac{f_{h^{ \pm}}^{2}}{8 \pi^{2}} \frac{m_{\mu}^{2}}{m_{h^{ \pm}}^{2}} \times\left(-\frac{1}{6}\right) .
$$

Hence from the Table 1, in the limit of $\theta=0$, the total contribution of singly charged Higgs is

$$
\Delta a_{\mu}^{\mathrm{E} 331}\left(h^{ \pm}\right)=-\left(\frac{1}{6}\right) \frac{\sqrt{2} G_{\mu} m_{\mu}^{2}}{4 \pi^{2}} \times \frac{m_{\mu}^{2}}{m_{H_{2}^{+}}^{2}} \frac{m_{Y}^{2}}{m_{Y}^{2}+m_{W}^{2}},
$$

where $m_{H_{2}^{+}}^{2}=\frac{2 \lambda_{4}}{g} m_{Y}^{2}[10]$.

Similarly to the case of neutral Higgs, the contribution of charged Higgs $\mathrm{H}_{2}^{+}$is of the order $10^{-10} \times \frac{1}{m_{H_{2}^{2}}^{2}}$ and negative therefore it will cancel out with the contribution of neutral Higgs.

The dominant contribution to $\Delta a_{\mu}^{N P}$ in the E331 framework is

$$
\Delta a_{\mu}^{N P}=a_{\mu}\left(Y^{+}\right)+a_{\mu}\left(Z^{\prime}(f)\right)+\left[a_{\mu}(Z(f))+193.89 \times 10^{-11}\right] .
$$

It is very interesting that values of contributions to $a_{\mu}$ in (31) depend on only $m_{Y}^{+}$, therefore this quantity gives a very good constraint on the values of $m_{Y}$, i.e value of $\mathrm{SU}(3)_{L}$ breaking scale. The numerical result is shown in Fig. 2. It is easy to see that the total contribution from the E331
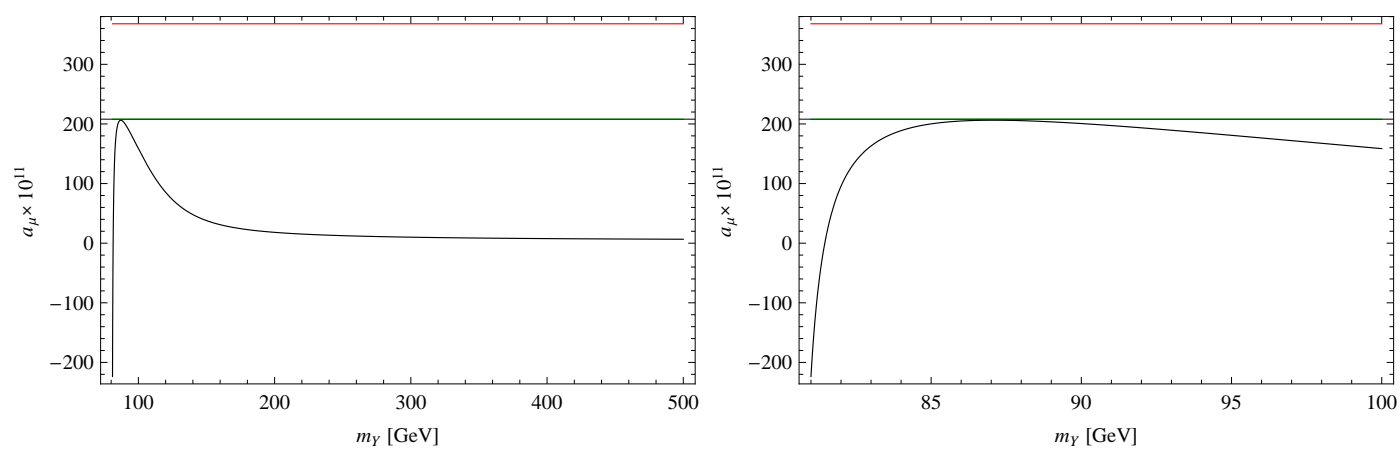

Fig. 2. Contributions of gauge bosons to $\Delta a_{\mu}^{N P}$

effects does not enough to explain the experimental results of $a_{\mu}$. For other 3-3-1 versions, there may be some new contributions from new particles such as the exotic heavy charged (neutral) leptons or more new charged gauge bosons may increase the values of $\Delta a_{\mu}^{331}$. We will comment more details in the section V. 


\section{COMMENTS ON CONSTRAINT OF SU $(3)_{L}$ SCALE FROM $a_{\mu}$ IN OTHER 3-3-1 MODELS}

\section{V.1. The reduced minimal 3-3-1 models (RM331)}

Coming back to investigation in Ref. [7]. The contributions to $\Delta a_{\mu}^{N P}$ are modified as follows

- From $V^{+}$gauge boson. The formula (31) in Ref. [7] is corrected as

$$
\Delta a_{\mu}\left(V^{+}\right)=a^{S M}(W) \times \frac{m_{W}^{2}}{m_{V^{+}}^{2}} .
$$

- Contribution from the $Z^{\prime}$ boson is rewritten in the explicitly form

$$
\Delta a_{\mu}^{R M 331}\left(Z^{\prime}\right)=-a_{\mu}^{S M}(W) \times \frac{m_{Z}^{2}}{m_{Z^{\prime}}^{2}} \times \frac{3\left(1-4 s_{W}^{2}\right)}{20} .
$$

- Contribution from $U^{++}$boson are written as

$$
a_{\mu}^{R M 331}\left(U^{++}\right)=a_{\mu}^{S M}(W) \times \frac{42}{5} \times \frac{m_{W}^{2}}{m_{U^{++}}^{2}} .
$$

Remember that $m_{U^{++}}^{2}=m_{W}^{2}+m_{V^{+}}^{2}$ and $m_{Z^{\prime}}^{2}=\frac{4 c_{W}^{2}}{3\left(1-4 s_{W}^{2}\right)} m_{V+}^{2}$ so the total contribution to $a_{\mu}$ in the RM331 limit, namely $\Delta a_{\mu}^{R M 331}=a_{\mu}\left(V^{+}\right)+a_{\mu}^{R M 331}\left(Z^{\prime}\right)+a_{\mu}^{R M 331}\left(U^{++}\right)$, depends on only the $m_{V^{+}}$ that is characterized for $S U(3)_{L}$ scale. The Fig. 3 shows the numerical result.
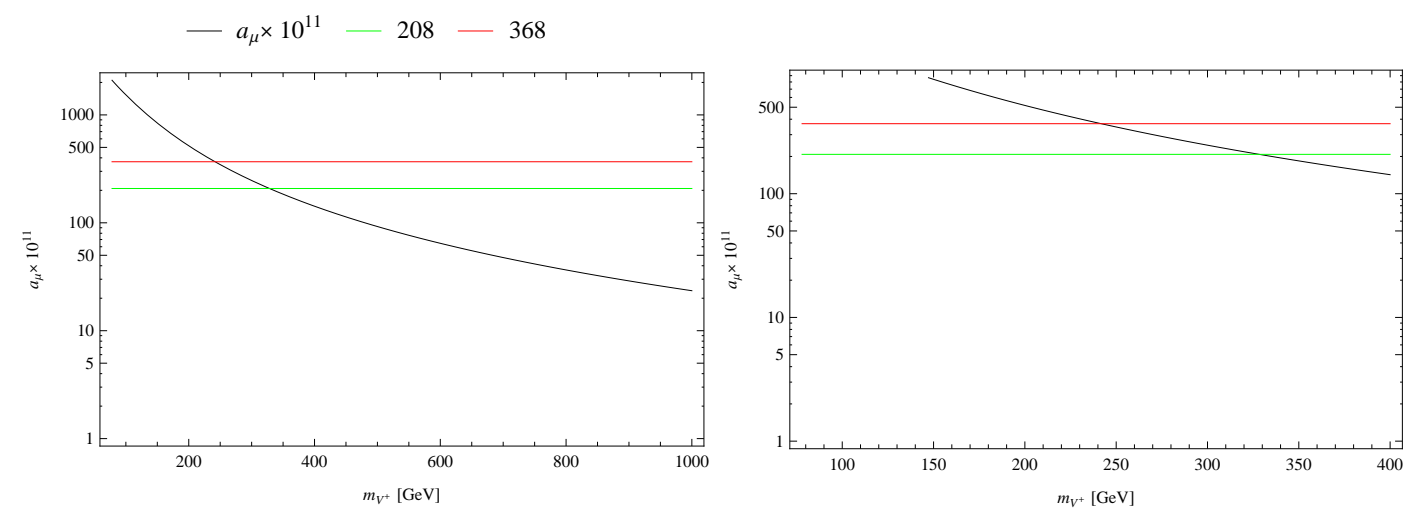

Fig. 3. Contribution of gauge bosons to $a_{\mu}$ in the RM331 model.

\section{V.2. The 3-3-1 model with exotic heavy lepton}

Some other models containing exotic heavy leptons which may give large contribution to $a_{\mu}$, such as [4] for new neutral leptons.

For the 3-3-1 model shown in [4], the $W^{+}$boson couples with left-handed neutrinos exactly the same as that in the SM while it does not couples with heavy neutrinos. In opposite, the new singly charged gauge boson $V^{+}$couples directly with heavy right-handed neutrinos $N_{R}$ with 
coupling $\frac{g}{2 \sqrt{2}}\left(1-\gamma_{5}\right)$. Hence the new contribution is from only this new $V^{+}$with value determined based on (17), in specially

$$
\Delta a_{\mu}\left(V^{ \pm}\right)=a_{\mu}^{S M}(W) \frac{3 m_{W}^{2}}{10 m_{V^{+}}^{2}} \int_{0}^{1} d x \frac{F^{V}(x)+F^{A}(x)}{\varepsilon^{2} \lambda^{2}(1-x)\left(1-\varepsilon^{-2} x\right)+x}
$$

where $\varepsilon=\frac{m_{N_{R}}}{m_{\mu}}$, and $\lambda=\frac{m_{\mu}}{m_{V^{+}}}$. In this case where $\varepsilon \gg 1$ and $\lambda \ll 1$ we get

$$
\begin{aligned}
\Delta a_{\mu}\left(V^{+}\right) & \simeq a_{\mu}^{S M}(W) \frac{3 m_{W}^{2}}{10 m_{V^{+}}^{2}} \int_{0}^{1} d x \frac{4 x^{2}(x+1)-2 \lambda^{2} \varepsilon^{2} x(1-x)(x+2)}{\varepsilon^{2} \lambda^{2}(1-x)+x} \\
& \equiv a_{\mu}^{S M}(W) \frac{3 m_{W}^{2}}{10 m_{V^{+}}^{2}} f(k)
\end{aligned}
$$

with $k=m_{N_{R}}^{2} / m_{V^{+}}^{2}$. Numerical values of $\mathrm{f}(\mathrm{k})$ are estimated in the Fig. 4.
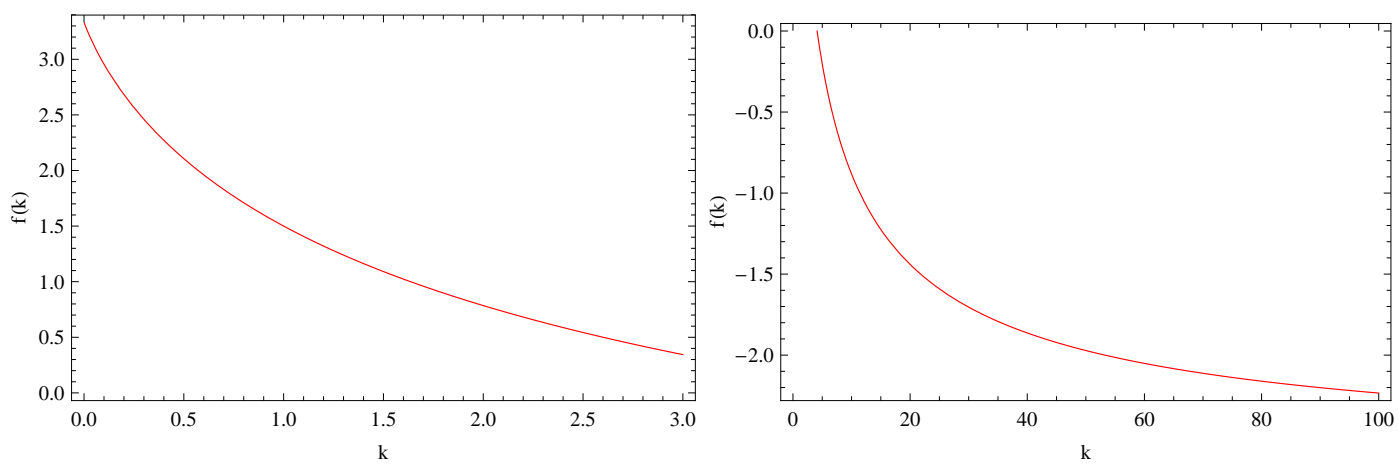

Fig. 4. The $f(k)$ values as functions of $k=m_{N_{R}}^{2} / m_{V^{+}}^{2}$ in two cases: $k<1$ (left panel) and $k>1$ (right panel).

It can be seen that $f(k)$ is always equivalent to $\mathscr{O}(1)$ so $\Delta a_{\mu}\left(V^{+}\right)$is always smaller than $a_{\mu}^{S M}(W)$ by a factor of $\frac{3 m_{W}^{2}}{10 m_{V^{+}}^{2}}$. Hence $m_{V^{+}}^{2}$ has to be order of $m_{W}^{2}$ as $\Delta a_{\mu}\left(V^{+}\right)$has order of $\Delta a_{\mu}^{N P}$. The case is similar to the doubly charged gauge bosons appearing in the minimal 3-3-1 model.

\section{CONCLUSIONS}

We have performed all leading-order contributions to the muon anomalous magnetic moment in the economical 3-3-1 model. In the E331 framework, our calculation has indicated that the electroweak one-loop contributions to $a_{\mu}$ are not large enough to explain the value given by recent experimental result, if the mass of the singly charged gauge boson is significant larger than that of the $W$ boson, i.e., the $S U(3)_{L}$ breaking scale is much larger than the electroweak breaking scale, as usually supposed in literature. If all of the scalars are heavy than $100 \mathrm{GeV}$, the contribution from gauge bosons are always dominant, especially the charged gauge bosons. So the model needs to be extended to have new gauge bosons coupling with charged leptons. The case is similar to other versions with right-handed neutrinos where there exists a neutral gauge boson that does not couple with charged lepton. For the case of the minimal 3-3-1 model, base on the calculation in Ref. [7] we have shown that, although there are more contributions from the doubly charged 
MUON ANOMALOUS MAGNETIC MOMENT IN E331 MODEL

gauge bosons, the total contribution is not still large enough to put the $S U(3)_{L}$ scale toward the $\mathrm{TeV}$ values. Supersymmetric version of the E331 model or other 331 models may provide a good solution for the $a_{\mu}$ results by the contributions from the superpartners.

\section{ACKNOWLEDGEMENTS}

This research is funded by the Vietnam National Foundation for Science and Technology Development (NAFOSTED) under grant number 103.01-2014.51.

\section{REFERENCES}

[1] J. Beringer et al., (Particle Data Group), Phys. Rev. D 86 (2012) 010001.

[2] J. P. Miller, E. de Rafael, B. L. Roberts, Rept. Prog. Phys. 70 (2007) 795, [hep-ph/0703049];

[3] M. Endo, K. Hamaguchi, T. Kitahara, T. Yoshinaga, JHEP 1311 (2013) 013,[arXiv:1309.3065]; M. Ibe, T. T. Yanagida, N. Yokozaki, JHEP 1308 (2013) 067, [arXiv:1303.6995]; L. L. Everett, G. L. Kane, S. Rigolin, L.-T. Wang, Phys. Rev. Lett. 86 (2001) 3484, [hep-ph/0102145]; R. L. Arnowitt, B. Dutta, B. Hu, [hep-ph/0310103]; S. P. Martin, J. D. Wells, Phys. Rev. D 67 (2003) 015002, [hep-ph/0209309].

[4] H. N. Long, Phys. Rev. D 54 (1996) 4691, [hep-ph/9607439]; F. Pisano, V. Pleitez, Phys. Rev. D 46 (1992) 410, [hep-ph/9206242]; R. Foot, H. N. Long, T. A. Tran, Phys. Rev. D 50 (1994) R34, [hep-ph/9402243]; C. A. de S. Pires, P. S. Rodrigues da Silva, JCAP 0712 (2007) 012, [arXiv:0710.2104]; J. K. Mizukoshi, C. A. de S. Pires, F. S. Queiroz, P. S. R. da Silva, Phys. Rev. D 83 (2011) 065024, [arXiv:1010.4097]; F. Queiroz, C. A. de S. Pires, P. S. R. da Silva, Phys. Rev. D 82 (2010) 065018, [arXiv:1003.1270]; D. Cogollo, A. V. de Andrade, F. S. Queiroz and P. Rebello Teles, Eur. Phys. J. C 72 (2012) 2029, [arXiv:1201.1268]; J. D. RuizAlvarez, C. A. de S.Pires, F. S. Queiroz, D. Restrepo and P. S. Rodrigues da Silva, Phys. Rev. D 86 (2012) 075011, [arXiv:1206.5779]; J. E. C. Montalvo, R. J. G. Ramrez, G. H. R. r. Ulloa and A. I. R. Mendoza, [arXiv:1311.0845]; A. Alves, E. Ramirez Barreto, A. G. Dias, C. A. de S.Pires, F. S. Queiroz and P. S. Rodrigues da Silva, Phys. Rev. D 84 (2011) 115004, [arXiv:1109.0238]; A. Doff and A. A. Natale, Int. J. Mod. Phys. A 27 (2012) 1250156, [arXiv:1210.3390]; A. Alves, E. Ramirez Barreto, A. G. Dias, C. A. de S.Pires, F. S. Queiroz and P. S. Rodrigues da Silva, Phys. Rev. D 84 (2011) 115004 [arXiv:1109.0238]; S. Profumo and F. S. Queiroz, arXiv:1307.7802; C. Kelso, C. A. d. S. Pires, S. Profumo, F. S. Queiroz and P. S. R. da Silva, [arXiv:1308.6630]; F. S. Queiroz,[arXiv:1310.3026]. A. Alves, E. Ramirez Barreto, A. G. Dias, C. A. de S.Pires, F. S. Queiroz and P. S. Rodrigues da Silva, Eur. Phys. J. C 73 (2013) 2288, [arXiv:1207.3699]; P. V. Dong, H. T. Hung, T. D. Tham , Phys. Rev. D 87 (2013) 115003, [arXiv:1305.0369]; P. V. Dong, T. Phong Nguyen, D. V. Soa, [arXiv:1308.4097]; J. G. Ferreira, C. A. d. S. Pires, P. S. R. da Silva and A. Sampieri, Phys. Rev. D 88, 105013 (2013), [arXiv:1308.0575]; J. M. Cabarcas, J. Duarte and J. -A. Rodriguez, [arXiv:1310.1407].

[5] P. V. Dong, H. N. Long, D. T. Nhung and D. V. Soa, Phys. Rev. D 73, 035004 (2006).

[6] C.A. de S. Pires, P. S. Rodrigues da Silva, Phys. Rev. D 64 (2001) 117701, [hep-ph/0103083]; C. A. De Sousa Pires, P. S. Rodrigues da Silva, Phys. Rev. D 65 (2002) 076011, [hep-ph/0108200]; N. A. Ky, H. N. Long, D. V. Soa, Phys. Lett. B 486 (2000) 140, [hep-ph/0007010]; N. A. Ky, H. N. Long, [hep-ph/0103247].

[7] C. Kelso, P. R. D. Pinheiro, F. S. Queiroz, and W. Shepherd, Eur. Phys. J. C 74 (2014) 2808

[8] P. V. Dong, H. N. Long and H. T. Hung, Phys. Rev. D 86 (2012) 033002, [arXiv:1205.5648].

[9] P.V. Dong, H. N. Long, D.V. Soa, Phys. Rev. D 75 (2007) 073006.

[10] P. V. Dong, H. N. Long, Adv. High Energy Phys. 2008 (2008) 739492, [arXiv:0804.3239(hep-ph)].

[11] T. Kinoshita and M. Nio, Phys. Rev. D 73 (2006) 013003;T. Aoyamaet al., Phys. Rev. Lett. 99 (2007) 110406; T. Kinoshita and M. Nio, Phys. Rev. D 70 (2004) 113001; T. Kinoshita, Nucl. Phys. B 144 (2005) 206 (Proc. Supp.); T. Kinoshita and M. Nio, Phys. Rev. D 73 (2006) 053007; A. L. Kataev, arXiv:hep-ph/0602098(2006); M. Passera, J. Phys. G 31 (2005) 75.

[12] G. Gabrielse et al., Phys. Rev. Lett. 97 (2006) 030802;Erratumibid. Phys. Rev. Lett. 99 (2007) 039902; D. Hanneke, S. Fogwell, and G. Gabrielse, Phys. Rev. Lett. 100 (2008) 120801.

[13] R. Jackiw and S. Weinberg, Phys. Rev. D 5, 2396 (1972);G. Altarelli et al., Phys. Lett. B 40 (1972) 415; I. Bars and M. Yoshimura, Phys. Rev. D 6 (1972) 374; K. Fujikawa, B. W. Lee, and A. I. Sanda, Phys. Rev. D 6 (1972) 2923. 
[14] G. Degrassi and G. F. Giudice, Phys. Rev. D 58 (1998) 053007.

[15] J. Bijnens and J. Prades, Mod. Phys. Lett. A 22, 767(2007); K. Melnikov and A.Vainshtein, Phys. Rev. D 70, 113006 (2004). M. Knecht and A. Nyffeler, Phys. Rev. D 65 (2002) 073034; M. Knechtet al., Phys. Rev. Lett. 88 (2002) 071802; J. Bijnens et al., Nucl. Phys. B 626 (2002) 410; J. Prades, E. de Rafael, and A. Vainshtein, Advanced series on directions in high energy physics 20, Editors B. L. Roberts and W. Marciano, arXiv:0901.0306[hepph] (2009). J. Hayakawa and T. Kinoshita, Erratum Phys. Rev. D 66 (2002) 019902; E. de Rafael, Phys. Lett. B 322 (1994) 239.

[16] G. W. Bennett et al., Phys. Rev. Lett. 89 (2002) 101804; Erratum Phys. Rev. Lett. 89 (2002) 129903; G. W. Bennett et al., Phys. Rev. Lett. 92 (2004) 161802; G. W. Bennett et al., Phys. Rev. D 73 (2006) 072003.

[17] R. M. Carey, K. R. Lynch, J. P. Miller, B. L. Roberts, W. M. Morse, Y. K. Semertzides, V. P. Druzhinin and B. I. Khazin et al., FERMILAB-PROPOSAL-0989.

[18] T. Blum, A. Denig, I. Logashenko, E. de Rafael, B. L. Roberts, T. Teubner, G. Venanzoni, [arXiv:1311.2198]; Y. Alexahin et al., [arXiv:1308.2143]; T. Blum et al.,[arXiv:1310.6087].

[19] P. V. Dong, H. N. Long and D. V. Soa, Phys. Rev. D 75 (2007) 073006.

[20] F. Jegerlehner, A. Nyffeler, Phys. Reports 477 (2009) 1, [arXiv:0902.3360].

[21] R. Jackiw, S. Weinberg, Phys. Rev. D 5 (1972) 2396; I. Bars, M. Yoshimura, Phys. Rev. D 6 (1972) 374; G. Altarelli, N. Cabibbo, L. Maiani, Phys. Lett. B 40 (1972) 415; W. A. Bardeen, R. Gastmans, B. Lautrup, Nucl. Phys. B 46 (1972) 319; K. Fujikawa, B. W. Lee, A. I. Sanda, Phys. Rev. D 6 (1972) 2923.

[22] V. Pleitez and M. D. Tonasse, Phys. Rev. D 48 (1993) 2353 